\title{
Optimal Temperature Control of a Steady-State Exothermic Plug-Flow Reactor
}

\author{
Ilse Y. Smets \\ Bioprocess Technology and Control (BioTeC), Katholieke Universiteit Leuven, B-3001 Leuven, Belgium \\ Denis Dochain \\ CESAME, Université Catholique de Louvain, B-1348 Louvain-la-Neuve, Belgium \\ Jan F. Van Impe \\ Bioprocess Technology and Control (BioTec), Katholieke Universiteit Leuven, B-3001 Leuven, Belgium
}

\begin{abstract}
Optimal heut exchanger temperature profiles of exothermic tubular reactors were determined under the assumption of steady-state and plug-flow characteristics. The minimum principle of Pontryagin (optimal control theory) was applied in a straightforward analytical sense. To enable a trade-off between process performance and heat loss, a combined cost criterion was defined. In the first approach of specifying only terminal costs, the optimal control input was of the bang-bang type that keeps the heat exchanger temperature constant at its maximum or minimum value. Aftenwards, the terminal cost criterion was extended with an integral part that accounts for the global heat loss during the process. This integral cost part induced a control of the bang-singular-bang type. The desired performance can be met by selecting appropriate weights for terminal and integral costs.
\end{abstract}

\section{Introduction}

Although tubular reactors have been largely used in the chemical process industries for several decades, the use of partial differential equation (PDE) models, not only for numerical simulation but also for system analysis and design of estimation and control algorithms has taken an increasing importance over the past decades (Christofides and Daoutidis, 1996, 1998a,b; Dochain, 2000; Gay and Ray, 1995; Winkin et al,, 2000; Ydstie and Coffey, 1998), and fairly recently (Christofides, 2001) following the pioncering works of, among others, Ray (1978, 1981) and Varma and Aris (1977). The question that we would like to address in this article is the determination of optimal profiles in nonisothermal tubular reactors. This work is basically motivated by our need to select a desired (optimal) profile or profiles that would be appropriate in the application of controllers whose design is based on a PDE model of the process.

In a first step, nonisothermal tubular reactors, in which convective phenomena dominate diffusive and dispersive mechanisms, are studied. The resulting equations are quasilinear (weakly) hyperbolic first-order PDEs describing reactors with plug-flow characteristics. In steady state, these re-

Correspondence concorning this article should be addrensed to J. F. Van Impe. duce to ordinary differential equations where the independent variable is the spatial variable $z$. More specifically, we are looking for the optimal solution with respect to a defined cost function of a set of two nonlinear differential equations where the input is the heat exchanger temperature and the output is the reactor temperature.

The problem results in an optimal control problem where the usual independent variable (the time $t$ ) is replaced by the spatial variable $z$. Different cost functions have been considered in our study. The first objective is to minimize the concentration of the reactant at the end of the reactor which is a measure for the process performance. This terminal cost is then extended with a sccond terminal cost that penalizes the deviation of the exit temperature $T$ from the inlet temperature $T_{\text {in }}$ to minimize the heat loss. Finally, in order to minimize the glohal heat loss, an integral cost is added to maintain the temperature $T$ within the reactor as close as possible to the inlet flow temperature $T_{\text {in }}$.

\section{Tubular Reactor Model}

In this study, plug-flow reactors are considered in which convection phenomena dominate over diffusive and disper- 
sive phenomena. In transient conditions, the system equations are quasi-linear first-order (weakly) hyperbolic PDEs. The distinct feature of hyperbolic PDEs is that all the eigenmodes of the spatial differential operator contain the same, or nearly the same, amount of energy, and thus an infinite number of modes is required to accurately describe their dynamic behavior (Christofides and Daoutidis, 1998a).

\section{Steady-state plug-flow equations with dimensionless variables}

To compensate for the large differences in the order of magnitude of the model variables (possibly resulting in numerical simulation inaccuracies), the following dimensionless state variables $x_{1}, x_{2}$, and dimensionless control input $u$, are introduced

$$
x_{1} \stackrel{ \pm}{=} \frac{T-T_{\mathrm{in}}}{T_{\mathrm{in}}}, \quad x_{2} \stackrel{ \pm}{=} \frac{C_{\mathrm{in}}-C}{C_{\mathrm{in}}}, \quad u \stackrel{ \pm}{=} \frac{T_{n}-T_{\mathrm{in}}}{T_{\mathrm{in}}}
$$

When defining the constants $\alpha, \beta, \gamma$ and $\delta$ as follows:

$$
\alpha \stackrel{د}{=} k_{0} e^{-E / R T_{\text {in }}}, \quad \beta \stackrel{\Delta}{=} \frac{4 h}{\rho C_{p} d}, \quad \delta \stackrel{د}{=}-\frac{\Delta H}{\rho C_{p}} \frac{C_{\text {in }}}{T_{\text {in }}}
$$

and

$$
\gamma \stackrel{ \pm}{=} \frac{E}{R T_{\text {in }}}
$$

the standard steady-state plug-flow equations with first-order Arrhenius type kinetics become

$$
\begin{array}{ll}
\frac{d x_{1}}{d z}=\frac{\alpha \delta}{v}\left(1-x_{2}\right) e^{\gamma x_{1} / 1+x_{1}}+\frac{\beta}{v}\left(u-x_{1}\right) & x_{1}(0)=0 \\
\frac{d x_{2}}{d z}=\frac{\alpha}{v}\left(1-x_{2}\right) e^{\gamma x_{1} / 1-x_{1}} & x_{2}(0)=0
\end{array}
$$

\section{Simulations with constant heat exchanger temperature}

When applying a constant heat exchanger temperature profile $T_{n}$, the temperature in the reactor $T(z)$ and the reactant concentration $C(z)$ behave as plotted in Figure 1. The values of the parameters and operating conditions are in spired by the values in Fjeld and Ursin (1971) (the value of the superficial fluid velocity $v$ has been changed from 0.04 $\mathrm{m} / \mathrm{s}$ to $0.1 \mathrm{~m} / \mathrm{s}$ and the value for $\delta$ has been changed from 0.59 to 0.25 ) and are depicted in Table 1 . Observe that, if a low heat exchanger temperature is chosen (such as, $T_{n}=300$ $\mathrm{K})$, the reactor temperature monotonically decreases resulting in an inefficient conversion of the reactant $C$.

\section{Extremal Control Profile Determination}

\section{Statement of the optimal control problem}

The optimal control problem studied in this article is to find an admissible control $u^{*}(z) \in \mathcal{U}$ which causes system Eq. 1 to follow an admissible trajectory $x^{*} \in \mathfrak{X}$ (with the process state $\boldsymbol{x}$ being composed of the state variables $x_{1}$ and $x_{2}$ ), while at the same time minimizing following performance
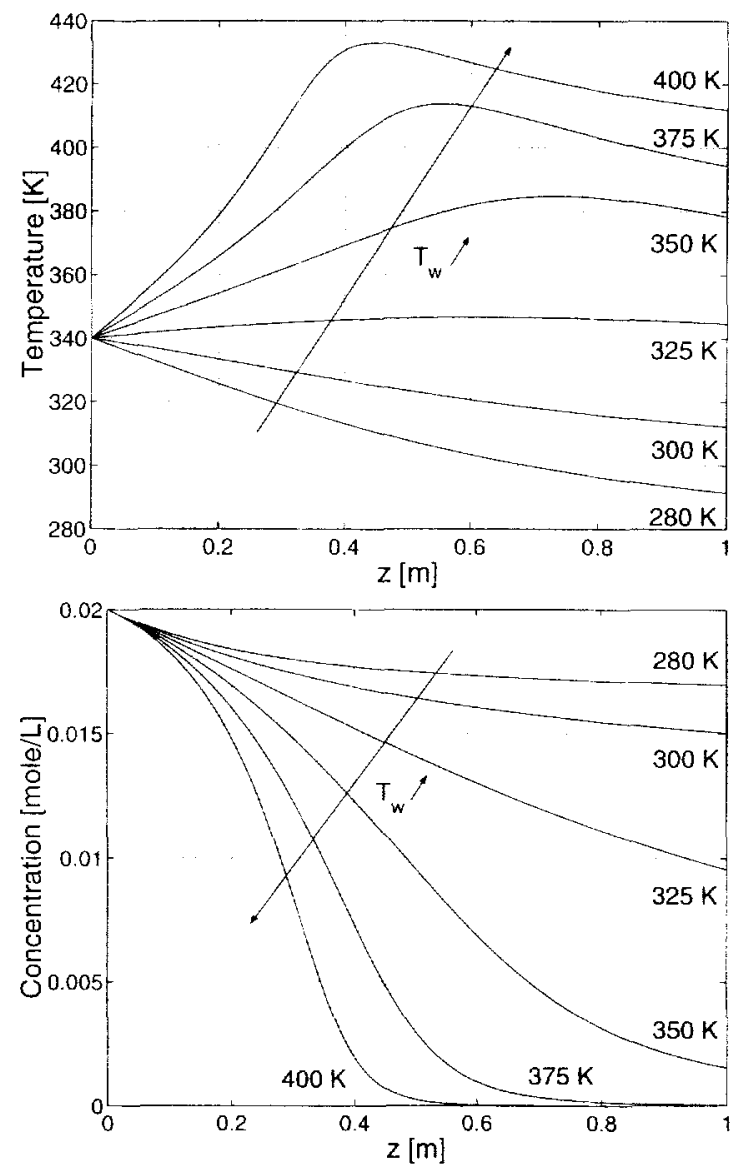

Figure 1. Temperature (upper plot) and concentration profiles (lower plot) for constant heat exchanger temperature $T_{w}$.

criterion (Kirk, 1970)

$$
\mathfrak{I}[u]=\underbrace{h[x(L)]}_{\text {Terminal cost }}+\underbrace{\int_{0}^{L} g[x(z)] d z}_{\text {Integral cost }}
$$

Remark 1. We limit ourselves to the integral cost as formulated in Eq. 2 to slightly facilitate forthcoming analytical manipulations. A more general integral cost $g[x(z), u(z), z]$ takes into account explicit dependencies on the control variable $u(z)$ and position $z$.

Table 1. Process Parameters

\begin{tabular}{rlrl}
$\nu$ & $=0.1$ & $\mathrm{~m} \cdot \mathrm{s}^{-1}$ \\
$L$ & $=1$ & & $\mathrm{~m}$ \\
$\delta$ & $=0.25$ & & \\
$E$ & $=11,250$ & & $\mathrm{cal} \cdot \mathrm{mol}^{-1}$ \\
$k_{0}$ & $=10^{6}$ & & $\mathrm{~s}^{-1}$ \\
$\beta$ & $=0.2$ & & $\mathrm{~s}^{-1}$ \\
$C_{\text {in }}$ & $=0.02$ & & $\mathrm{~mol} \cdot \mathrm{L}^{-1}$ \\
$R$ & $=1.986$ & & $\mathrm{cal} \cdot \mathrm{mol}^{-1} \cdot \mathrm{K}^{-1}$ \\
$T_{\text {in }}$ & $=340$ & & $\mathrm{~K}$ \\
\hline
\end{tabular}

AIChE Journal 
Remark 2. The admissible state domain $X$ is determined by: $T(z)>0$ and $0<C(z)<C_{\text {in }}$ which induce $x_{1}(z)>-1$ and $0<x_{2}(z)<1$, respectively. The admissible control domain $\mathfrak{U}$ is determined by: $T_{w, \text { MIN }}<T_{w}<T_{w \text {, MAX }}$ which induces $u_{\text {MIN }}$ $<u(z)<u_{\text {MAX }}$.

\section{Hamiltonian and costate equations}

According to the Minimum Principle of Pontryagin, the Hamiltonian $\mathfrak{H C}$ for this problem is (Kirk, 1970)

$$
\begin{aligned}
\mathcal{H}=\left[g[x(z)]+\lambda_{1}[\right. & \left.\frac{\alpha \delta}{v}\left(1-x_{2}\right) e^{\gamma x_{1}\left(1+x_{1}\right)}-\frac{\beta}{v} x_{1}\right] \\
& \left.+\lambda_{2}\left[\frac{\alpha}{v}\left(1-x_{2}\right) e^{\gamma x_{1}\left(1-x_{1}\right)}\right]\right]+\left[\lambda_{1} \frac{\beta}{v}\right] u \\
& \stackrel{J}{=} \phi+\psi u
\end{aligned}
$$

with $\lambda_{1}$ and $\lambda_{2}$ the so-called costates associated to $x_{1}$ and $x_{2}$, respectively. Since the Hamiltonian does not explicitly depend on the spatial coordinate $z$, the Hamiltonian is constant when evaluated on an extremal trajectory. The costates satisfy the following dynamic equations (with $\lambda$ the vector of $\left[\lambda_{1}\right.$ $\left.\left.\lambda_{2}\right]^{T}\right): d \boldsymbol{\lambda} / d z=-\partial \mathcal{H} / \partial x$, with boundary conditions: $\lambda(L)=$ $\partial h /\left.\partial x\right|_{L}$.

\section{Extremal control determination}

Clearly, if the integral cost part is not an explicit function of $u$, the control variable $u$ appears linearly in the Hamiltonian $\mathfrak{F}$. As a result, the extremal control $u^{*}(z)$ depends on the value of $\psi$ as follows: (i) if $\psi>0$, then $u^{*}(z)=u_{\text {MIN }}$; (ii) if $\psi=0$ for $z \in\left[z_{i}, z_{i+1}\right]$, then $u^{*}(z)=u_{\text {sing }}(z)$; (iii) if $\psi<0$, then $u^{*}(z)=u_{\mathrm{MAX}}$. This is a control of the bang-singular-bang type. As mentioned before, $u_{\mathrm{MIN}}$ and $u_{\mathrm{MAX}}$ represent the lower and upper bound on the control input, respectively.

\section{Singular arc analysis}

On a singular interval $\left[z_{i}, z_{i+1}\right]$, we have that $\psi=0$. The singular control $u_{\text {sing }}(z)$ is then obtained by repeatedly differentiating $\psi$ with respect to $z$ until $u$ appears explicitly. After two successive differentiations, the following expression is obtained

$$
\begin{aligned}
u_{\text {sing }} & =x_{1}-\frac{\alpha \delta}{\beta}\left(1-x_{2}\right) e^{\gamma x_{1}, \lambda\left(+x_{1}\right)}+\frac{\varphi}{\beta} \\
& {\left[\frac{\frac{\partial g[x(z)]}{\partial x_{2}}-\frac{\partial^{2} g[x(z)]}{\partial x_{1} \partial x_{2}} \frac{d x_{2}}{d z}}{\frac{\partial g[x(z)]}{\partial x_{1}}\left(\frac{\gamma}{\left(1+x_{1}\right)^{2}}-\frac{2}{1+x_{1}}\right)}\right] }
\end{aligned}
$$

At this point, the following remarks can be formulated:

Remark 1. The singular control is not explicitly dependent on the costates $\lambda_{1}$ and $\lambda_{2}$.

Remark 2. In a generic case the denominator of the singular control (Eq. 4$)$ is different from zero along the singular arc. Since two successive differentiations are needed to compute $u_{\text {sing }}(z)$, this problem is a singular control problem of order 2.

Remark 3. In the limiting case that $g[\boldsymbol{x}(z)]$ is not explicitly dependent on the state $x_{1}, \lambda_{2}(z)$ will be equal to zero along the reactor. In that case, a singular are is impossible since the Hamiltonian (Eq. 3 ) reduces to $\mathcal{H C}=g\left[x_{2}(z)\right]$, which cannot be constant.

Remark 4. The singular control (Eq. 4) consists of two parts. The first part (that is, the first two terms) is independent of the cost function (Eq. 2), while the second part (that is, the last term) is largely influenced by the selection of the integrand $g[x(z)]$ in the cost function (Eq. 2).

Remark 5. When substituting singular control (Eq. 4) in the first state equation (Eq. 1), we obtain

$$
\frac{d x_{1}}{d z}=\left[\frac{\frac{\partial g[x(z)]}{\partial x_{2}}-\frac{\partial^{2} g[x(z)]}{\partial x_{1} \partial x_{2}} \frac{d x_{2}}{d z}}{\frac{\partial \partial^{2} g[x(z)]}{\partial x_{1}^{2}}-\frac{\partial g[x(z)]}{\partial x_{1}}\left(\frac{\gamma}{\left(1+x_{1}\right)^{2}}-\frac{2}{1+x_{1}}\right)}\right]
$$

From this, the following result is obtained:

Result 1. A sufficient condition for the reactor temperature to be constant along the singular arc is that the integrand $g[\boldsymbol{x}(z)\}$ does not depend explicitly on the second state $x,(z)$.

To illustrate the above mentioned general procedure of determining the optimal heat exchanger profile $u^{*}(z)$, different (combinations of) performance costs will be considered. In a first case study only terminal costs are taken into account. This will be illustrated with an example. In a second case study the combination of a terminal and an integral cost term is discussed and illustrated with an example as well.

\section{Case No. 1: Optimal Control with Terminal Cost Only}

In this section the objective is to determine the optimal heat exchanger temperature profile $u^{*}(z)$ for mass balance Eq. 1 which minimizes

$$
\mathcal{J}[u]=h[x(L)]
$$

\section{Hamiltonian and costate equations}

If no integral cost part is considered, Eq. 3 reduces to

$$
\begin{aligned}
\mathcal{H} & =\left[\lambda_{1}\left[\frac{\alpha \delta}{v}\left(1-x_{2}\right) e^{\gamma x_{1}\left(1+x_{1}\right)}-\frac{\beta}{v} x_{1}\right]\right. \\
& \left.+\lambda_{2}\left[\frac{\alpha}{v}\left(1-x_{2}\right) e^{\gamma x_{1}\left(1+x_{1}\right)}\right]\right]+\left[\lambda_{1} \frac{\beta}{v}\right] \cdot u \\
& \doteq \phi+\psi u .
\end{aligned}
$$

We now prove that in the case of only a terminal cost (Eq. 5), a singular arc cannot occur and the optimal control is of bang-bang type. 


\section{Singular arc analysis}

Recall that $\psi$ and its subsequent derivatives with respect to $z$ are equal to zero on a singular arc. In the case of a terminal cost (Eq. 5), $\lambda_{1}(z)$, as well as $\lambda_{2}(z)$, equal zero along the reactor. The former follows from the $\psi=0$ condition and the latter from the $d \psi / d t=0$ condition and the fact that, starting from a non-zero inlet concentration, the reactant's concentration cannot go to zero within finite reactor length. Hence, both costates and their first derivatives with respect to $z$ are identically equal to zero along the singular arc. For a Hamiltonian of the form (Eq. 6), this implies that subsequent derivation will never yield an expression for the singular control $u_{\text {sing }}$.

From the above, the following generic result is obtained.

Result 2. If the performance index is of the terminal cost type (Eq. 5), then the optimal heat exchanger temperature profile is of bang-bang type, that is, a singular are is not possible.

\section{Example}

According to Result 2, the optimal heat exchanger profile $u^{*}(z)$ switches between its maximum value $u_{\mathrm{MAX}}$ and its minimum value $u_{\mathrm{MIN}}$ (or, equivalently, the heat exchanger temperature $T_{n}^{*}(z)$ switches between $T_{w \cdot \operatorname{MAX}}$ and $T_{w, \text { MIN }}$ ). In the following, the optimal control sequence will be determined for a number of cost functions. During numerical simulations, the upper and lower bound on the heat exchanger temperature $T_{n}$, have been fixed to $T_{w \text { MAX }}=400 \mathrm{~K}$ and $T_{n, \text { MIN }}$ $=280 \mathrm{~K}$, respectively.

We consider the following terminal cost

$$
\mathfrak{J}[u]=(1-A) \underbrace{\left(1-x_{2}(L)\right)}_{\mathfrak{J}_{1}[u]}+\frac{A}{K_{1}} \underbrace{x_{1}^{2}(L)}_{\mathfrak{d}_{2}[u]} .
$$

When considering the definition of the dimensionless variables, it is clear that the minimization of the first term $\mathfrak{d}_{1}[u]$ ( $A=0$ ) represents the minimization of the outlet reactant concentration $C(L)$, while the minimization of the second term $g_{2}[u](A=1)$ drives the outlet reactor temperature $T(L)$ towards the inlet reactor temperature $T_{\text {in }}$ to minimize the loss of heat. $K_{1}$ is a user-defined weight to bring both costs in the same order of magnitude. We now examine the performance of the following sequence for the heat exchanger temperature profile. Consider a step profile which starts at $u(z)=u_{\text {MAX }}$ (or, equivalently, $T_{n}(z)=T_{w, \text { MAX }}$ ) for $0 \leq z \leq z_{1}$ and then switches to $u(z)=u_{\mathrm{MIN}}$ (or, equivalently, $\left.T_{n}(z)=T_{w . \text { MIN }}\right)$ for $z_{1} \leq z \leq L$. Observe that there is one degree of freedom, namely, the position $z_{1}$ at which the switch occurs. In addition, two limiting cases can be distinguished. If $z_{1}=0$, then the heat exchanger temperature is equal to $T_{n}(z)$ $=T_{w, \text { MIN }}$ for all values of $z$. If $z_{1}=L$, then the heat exchanger temperature is equal to $T_{w}(z)=T_{w \cdot \operatorname{MAX}}$ for all values of $z$.

Minimization of Outlet Reactant Concentration. First, we focus on the first part $\mathfrak{d}_{1}[u]$ of the cost function (Eq. 7). In other words, the weighting factor $A$ is set zero. This corresponds to the minimization of the outlet reactant concentration $J_{1}\left[T_{n}\right]=C(L)$. The behavior of $J_{1}\left[T_{w}\right]$ with respect to $z_{1}$ is plotted in Figure 2 by a dashed line. Observe that, in order

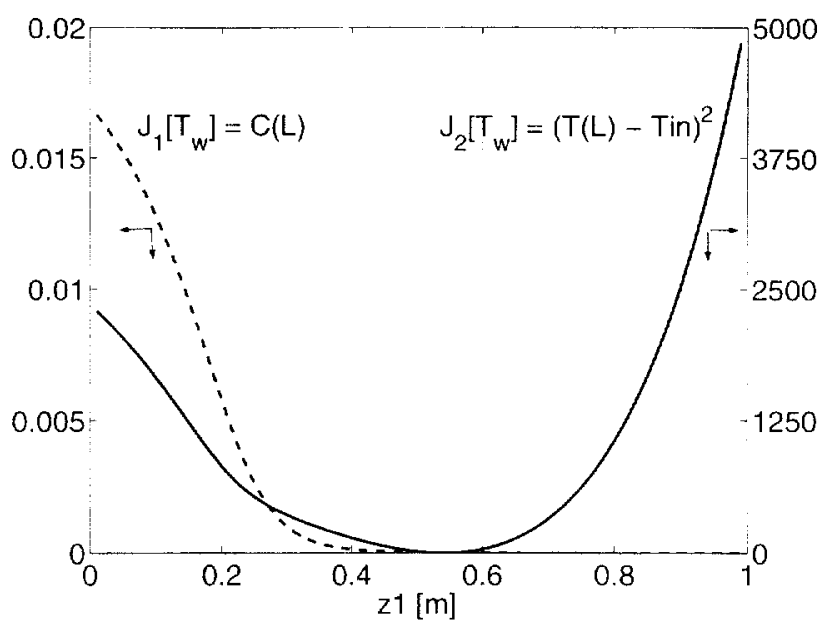

Figure 2. Maximum-minimum profile.

Cost criteria as function of switch position $z_{1} . J_{1}\left[T_{H}\right]=C(L)$ and $J_{2}\left[T_{11}\right]=\left(T(l)-T_{i n}\right)^{2}$.

to improve the interpretability of the results, in Figures $2-8$ the costs are shown using the original variables instead of the dimensionless variables (see also the figure captions). The following observations can be made:

(1) The cost reaches its minimum value at $z_{1}$ equal to $L$, that is, the optimal temperature profile is $T_{w}(z)=T_{w \cdot \text { MAX }}$ for all values of $z$. Notice that, as already mentioned, $C(L) e x-$ actly equal to zero cannot be reached within finite reactor length $L$.

(2) The optimal value ( $\left.z_{1}^{*}=L\right)$ obtained is not very pronounced: for values of $z_{1}$ larger than 0.4 , the outlet concentration is almost equal to zero.

(3) The corresponding optimal reactor temperature and concentration profiles are plotted in Figure $1\left(T_{n}=400 \mathrm{~K}\right)$. It can be seen that already half way up the reactor, the reactant is almost completely converted. Therefore, if minimization of the outlet reactant concentration is the only objective, the reactor length is not properly designed.

This leads to the following generic result.

Result 3. If the performance index is $\mathfrak{J} / u\}=1-x_{2}(L)$ lor, equivalently, $J\left[T_{w} /=C(L)\right]$, then the optimal control input is $u^{*}(z)=u_{\mathrm{MAX}}$, that is, the heat exchanger temperature profile is $T_{w^{*}}^{*}(z)=T_{w, \operatorname{MAX}}$ independent of the position $z$.

Minimization of Outlet Reactor Temperature. From Figure 1 , it can be seen that for $T_{n}(z)$ equal to $T_{w \cdot \text { MAX }}$ for all values of $z$, the outlet reactor temperature $T(L)$ is relatively high as compared to the inlet reactor temperature. In many practical applications, this may be undesirable. Therefore, we also consider optimization of a cost of type $J_{2}[u]$ in performance measure (Eq. 7).

The evolution of $J_{2}\left[T_{w}\right]=\left(T(L)-T_{\text {in }}\right)^{2}$ as a function of the degree of freedom $z_{1}$ is plotted in Figure 2, shown by a full line. At this point, the following remark can be formulated: The minimum value of $J_{2}\left[T_{n}\right]$ occurs at a switching position $z_{1}^{*}=0.54$. In other words, the optimal control is of the bangbang type. Indeed, for values of $z_{1}$ smaller than $z_{1}^{*}=0.54$, the cost $J_{2}\left[T_{w}\right]$ increases again. This can be explained as follows. If the switch occurs too early, the average heat exchanger temperature applied to the reactor is too low. This 


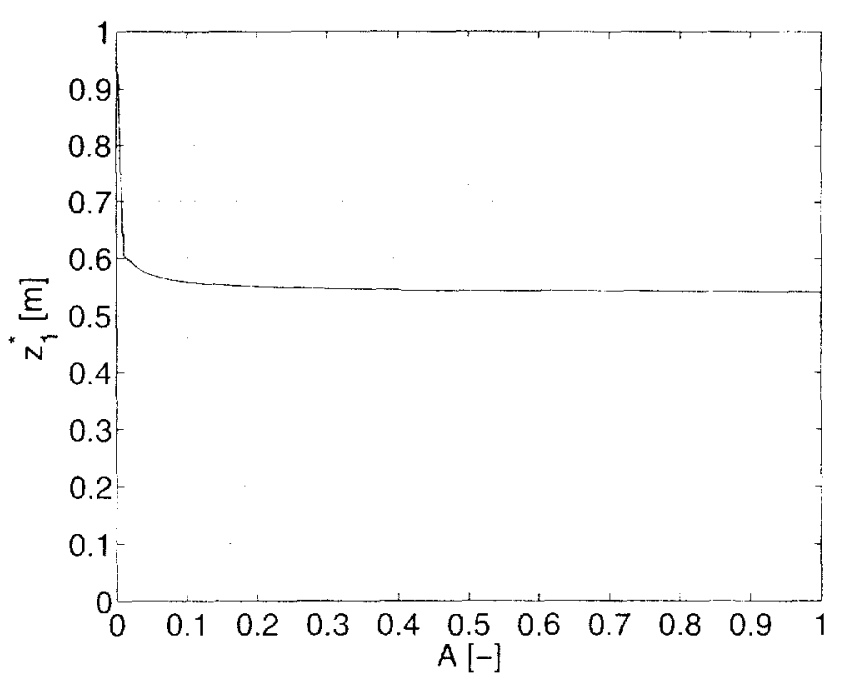

Figure 3. Optimization of $J\left[T_{w}\right]=(1-A) J_{1}\left[T_{w}\right]+$ $A / K_{1} J_{2}\left[T_{w}\right]$ : optimal switching position $z_{1}^{*}$ as function of the weight $A\left(K_{1}=250,000\right)$.

results in an outlet reactor temperature $T(L)$ smaller than $T_{\text {in. }}$. From Figure 2, it also can be immediately concluded that, for low values of $z_{1}$, the reactant conversion (dashed line) is very inefficient. The temperature and concentration profiles for the optimal switching position $z_{1}^{*}$ will be shown in Figure 4. Notice that the minimum value of the cost $J_{2}\left[T_{H}\right]$ is indeed reached: $T(L)=T_{\text {in }}$.

Weighted Minimization of Oullet Reactant Concentration and Outlet Reactor Temperature. In order to illustrate a weighted combination of the two terminal costs, the value of $A$ in Eq. 7 is varied between 0 and 1 . To bring both cost terms in the same order of magnitude, a value of 250,000 is assigned to $K_{1}$. As explained higher, if $A$ is equal to zero, then $J_{1}\left[T_{n}\right]$ is minimized and the minimum is reached at $z_{1}^{*}=1(=L)$. If $A$ is equal to one, $J_{2}\left[T_{H}\right]$ is the cost determining term in Eq. 7 and the minimum is reached at $z_{i}^{*}=0.54$. Therefore, generally speaking, the weighted cost $J\left[T_{w}\right]=(1-A) J_{1}\left[T_{w}\right]+$ $A / K_{1} J_{2}\left[T_{\mu}\right]$ will reach its minimum at some value $z_{1}^{*} \in$ $[0.54, \mathrm{~L}]$ for given value of $A$ in $[0,1]$. The optimal switching position $z_{1}^{*}$ as function of the weight $A$ is plotted in Figure 3.

It can be concluded that for most values of $A, J_{2}\left[T_{k i}\right]$ is the cost determining factor and $z_{i}^{*}$ remains (practically) equal to 0.54 . Therefore, the plots of Figure 4 also hold true for a value of $A$ equal to, say, 0.5 . In view of the minimization of the weighted cost $J\left[T_{n}\right]$, they can be interpreted as follows. The first half of the reactor length (more correctly, $0.54 \mathrm{~m}$ ) is used to reduce the reactant concentration $C(z)$ to very low values due to the maximum heat exchanger input. The second half of the reactor length (more precisely, $0.46 \mathrm{~m}$ ) is subsequently used to cool the reactor content to the target value of $T(L)=T_{\text {in }}$.

From this, the following result can be drawn.

Result 4. If the performance index is of the form Eq. 7, then the optimal control input $u^{*}(z)$ switches at most once from $u^{*}(z)=u_{\mathrm{MAX}}$ to $u^{*}(z)=u_{\mathrm{MIN}}$, that is, the heat exchanger temperature profile is a step profile starting at $T_{w}^{*}(z)=T_{n, \operatorname{MAx}}$ and switching (at most once) to $T_{w}^{*}(z)=T_{w, M I N}$.
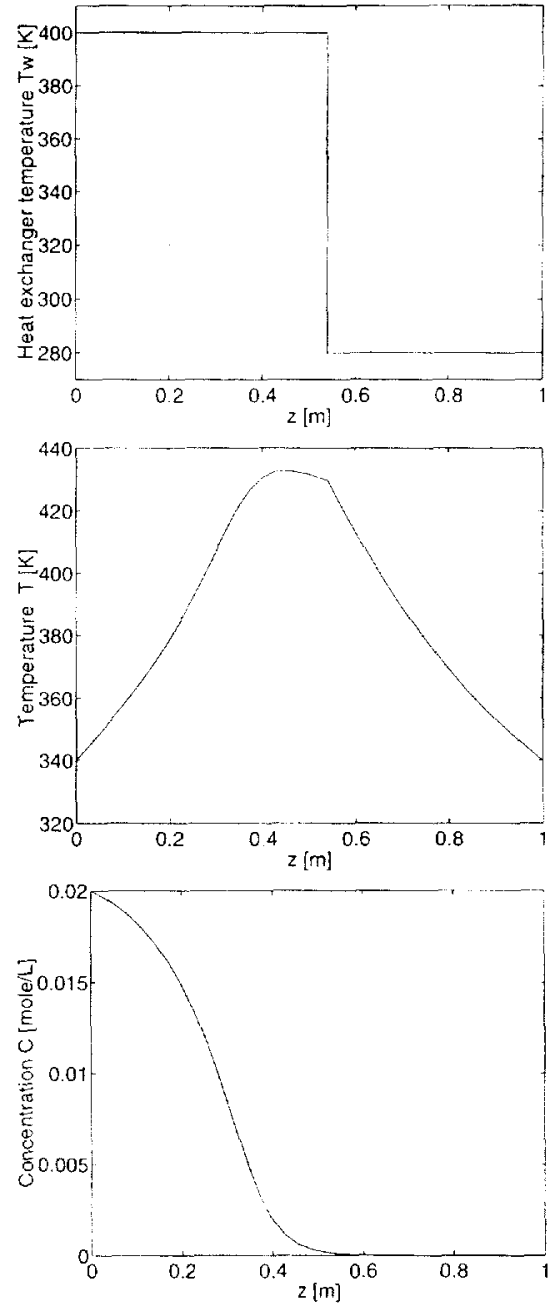

Figure 4. Maximum-minimum profile.

Optimization of $J_{2}\left[T_{11}\right]$. Optimal switching at $z_{i}^{*}=0.54 \mathrm{~m}$. Lpper: heat exchanger temperature profile. Middle: reactor temperature profilc. Bottom: reactant concentration profile.

\section{Case No. 2: Optimal Control with Integral and Terminal Cost}

In this section the performance criterion is of the general form (Eq. 2) with a terminal part and an integral part (with the latter assumed to be only a function of the states $\boldsymbol{x}(z)$ )

$$
\mathfrak{d}[u]=\underbrace{h[x(L)]}_{\text {Terminal cost }}+\underbrace{\int_{0}^{l} g[x(z)] d z .}_{\text {Integral cost }}
$$

The determination of the optimal heat exchanger temperature profile for this general cost will be illustrated with an example.

\section{Example}

Consider a cost criterion of the following type

$$
\mathfrak{d}[u]=(1-A) \underbrace{\left(1-x_{2}(L)\right)}_{\mathfrak{d}_{1}[u]}+\frac{A}{K_{2}} \int_{0}^{L} \frac{x_{1}^{2}(z) d z}{\mathscr{J}_{3}[u]}
$$


where $A$ is the trade-off coefficient between terminal and integral cost, and $K_{2}$ is a user-defined weighting factor to bring the two costs in the same order of magnitude. The terminal cost part is a measure for the process efficiency, while the integral cost part accounts for the total heat loss.

Singular Arc Analysis. Since the integral cost is not a function of the second state $x_{2}(z)$ expression Eq. 4 reduces to

$$
u_{\text {sing }}=x_{1}-\frac{\alpha \delta}{\beta}\left(1-x_{2}\right) e^{\gamma x_{1},\left(1+x_{1}\right)} \text {. }
$$

As mentioned before, this control keeps the temperature in the reactor constant along the singular arc. This is summarized in the following result:

Result 5. If the performance index is a sum of terminal and integral costs as in Eq. 9, then the optimal heat exchanger temperature profile $u^{*}(z)$ is of the bang-singular-bang type inducing a constant reactor temperature $T(z)$ during the singular arc.

The above analysis has shown that the control will be of the bang-singular-bang type. This is translated in a heat exchanger temperature profile moving from its maximum value $(400 \mathrm{~K})$ towards its minimum value $(280 \mathrm{~K})$ via a singular arc.

The profile, considered in this study, starts at $u(z)=u_{\text {MAX }}$ (or, equivalently, $\left.T_{n}(z)=T_{n} \cdot M \wedge X\right)$ for $0 \leq z \leq z_{1}$, subsequently switches to the singular control, that is, $u(z)=u_{\text {sing }}$ for $z_{1} \leq z \leq z_{2}$ and ends with $u(z)=u_{\text {MIN }}$ (or, equivalently, $\left.T_{n}(z)=T_{n, \text { MIN }}\right)$ for $z_{2} \leq z \leq L$. This will cause the temperature in the reactor to increase from the fixed inlet temperature $T_{\text {in }}$ (that is, $340 \mathrm{~K}$ ) towards the value $T^{*}$ at $z_{1}$ which will be kept constant until $z_{2}$ is reached, and will decrease afterwards. This heat exchanger temperature profile is presented in a qualitative way in Figure 5.

Maximum-Singular-Minimum Profile. This profile induces two degrees of freedom, namcly $z_{1}$, or, equivalently $T^{*}$ (that is, the value at which the temperature is kept constant along the singular arc), and $z_{2}$. After selection of $A$ and $K_{2}$, a hierarchic optimization scheme is adopted: given a value for $T^{*}$ (or, equivalently, $z_{1}^{*}$ ), the $z_{2}$ switching position is optimized in such a way that the global cost (Eq. 9) is minimized.

Figure 6 illustrates the behavior of the global cost $J\left[T_{w}\right]$ (Eq. 9) (full line) and its components $J_{1}\left[T_{w}\right]$ (dotted line) and

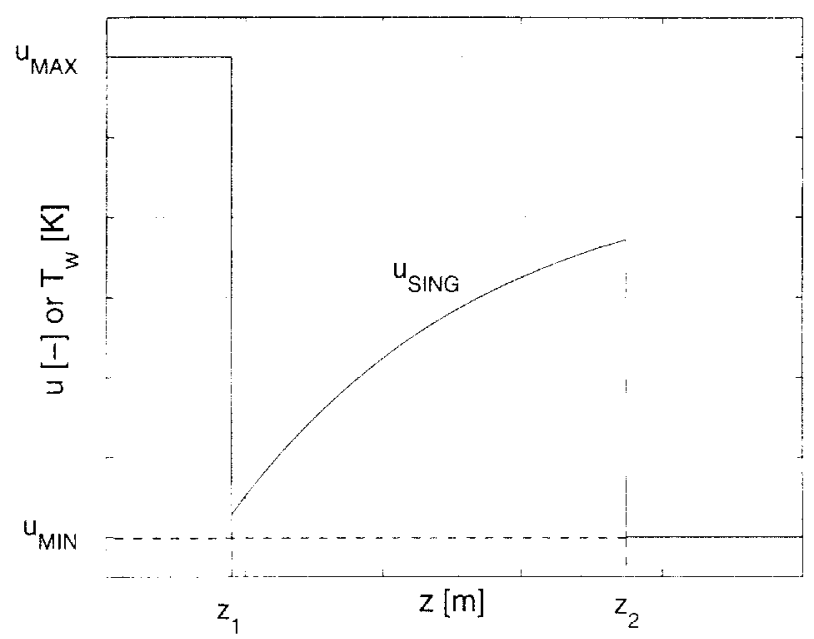

Figure 5. Maximum-singular-minimum profile.

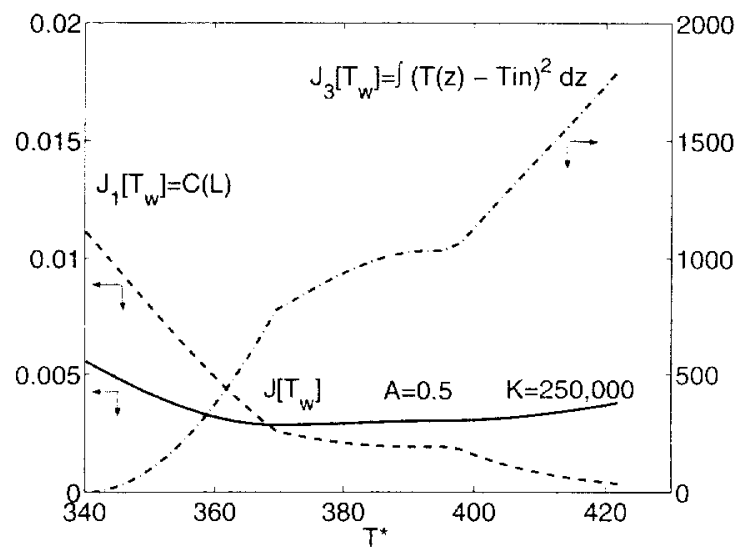

Figure 6. Maximum-singular-minimum profile.

Cost criteria as function of setpoint temperature $T^{*}, J_{1}\left[T_{n}\right]$ $=C(L), J_{3}\left[T_{H^{\prime}}\right]=\int_{0}^{L}\left(T(z)-T_{\text {in }}\right)^{2} d z$ and $J\left[T_{n}\right]=(1-$ A) $J_{1}\left[T_{w}\right]+A / K, J_{3}\left[T_{n}\right]$ with $A=0.5$ and $K_{2}=250.000$.

$J_{3}\left[T_{w}\right]$ (dashed-dotted line) as a function of the setpoint temperature $T^{*}$. The following observations can be made. First of all, a high $T^{*}$ value induces a high conversion of the reactant. This is similar to the results obtained with the maximum-minimum profile where a longer period of maximum heating increased the conversion. Secondly, the optimum $T^{*}$ value for the integral cost is, rather evidently, equal to $T_{\text {in }}$, that is, $340 \mathrm{~K}$.

For an equal weighting of the terminal and integral costs ( $A=0.5$ and $K_{2}=250,000$ ), the performance index $J\left[T_{k}\right]$ is globally optimized for $T^{*}=370 \mathrm{~K}$ (or, equivalently, $z_{1}^{*}=0.16$ $\mathrm{m})$.

Comparison of maximum-singular-minimum profile with maximum-minimum profile. Recall that the optimal profile for a cost criterion with only terminal cost parts is of bang-bang type, which has to be extended with a singular part if an integral cost part is included. Although optimality in the sense of the minimum principle cannot be guaranteed in the latter case, the maximum-minimum profile has attractive properties

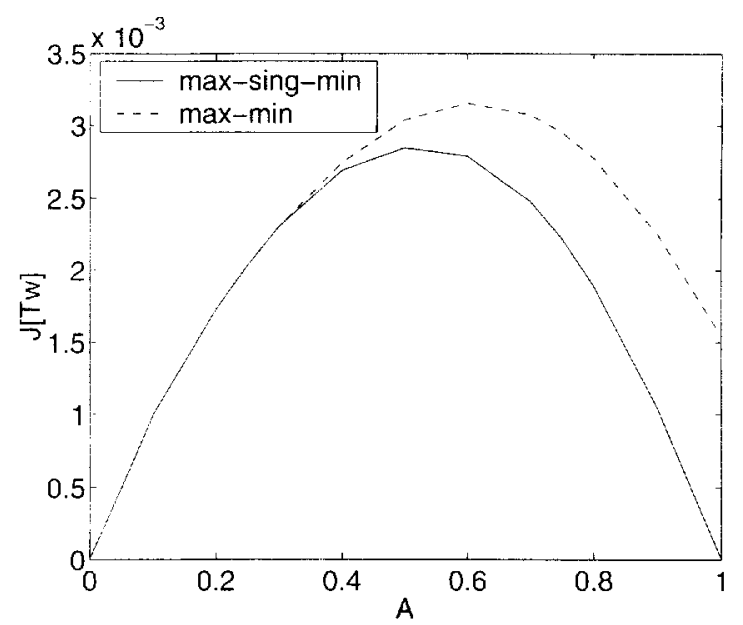

Figure 7. Maximum-singular-minimum profile (-) vs. maximum-minimum profile $(--)$ for $J\left[T_{w}\right]=$ $(1-A) J_{1}\left[T_{w}\right]+A / K_{2} J_{3}\left[T_{w}\right]$ with $K_{2}=250,000$. 

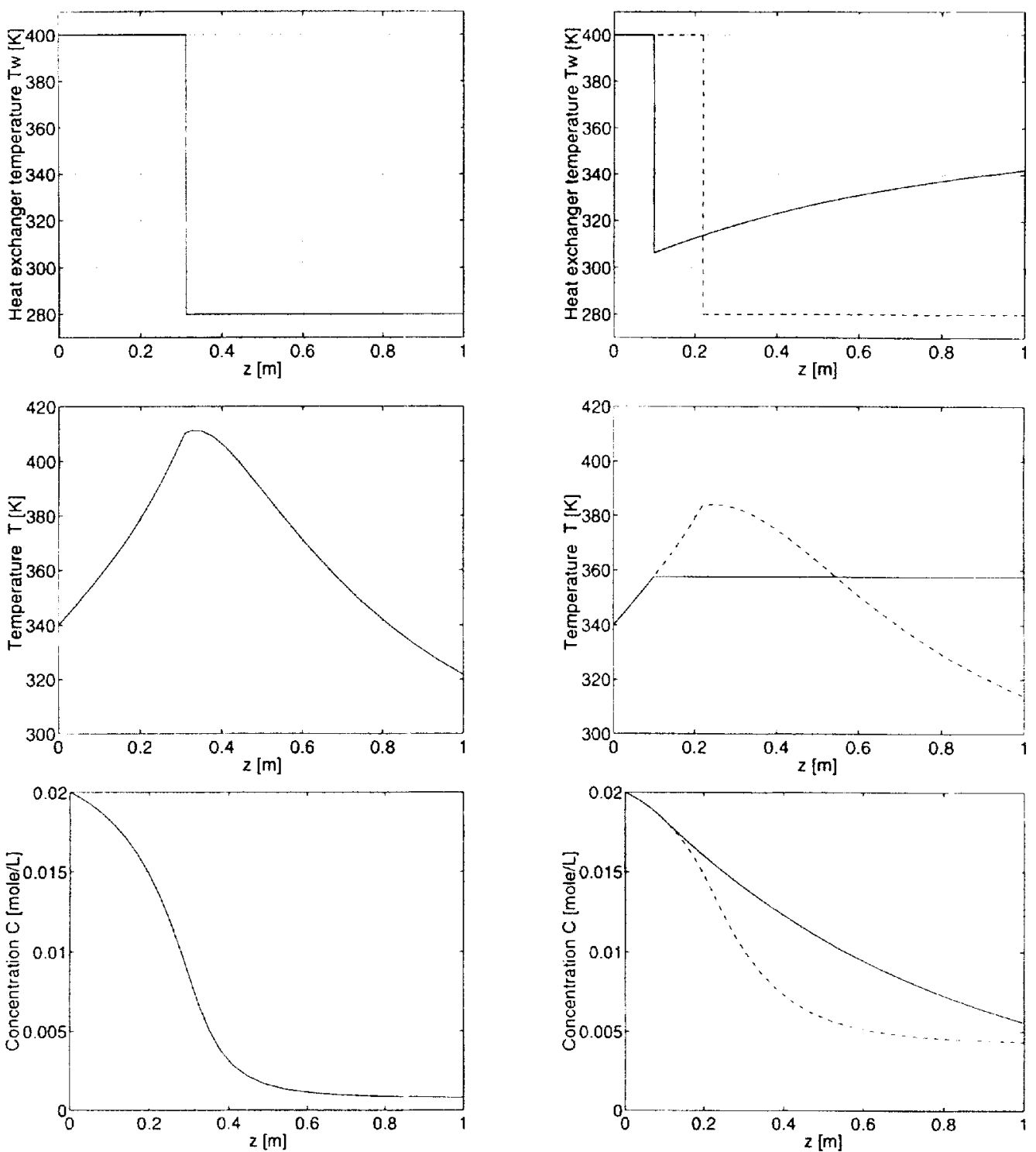

Figure 8. Maximum-singular-minimum profile (-) vs. maximum-minimum profile $(--)$ for $J\left[T_{w}\right]=(1-A) J_{1}\left[T_{w}\right]+$ $A / K_{2} J_{3}\left[T_{w}\right]$ with $K_{2}=250,000$ and $A=0.3$ (left plots) or $A=0.7$ (right plots).

Upper: heat exchanger temperature profiles. Middle: reactor temperature profiles. Bottoni: reactant concentration profiles.

with respect to practical implementation. The heat exchanger should consist of two parts (the one at $T_{w}=T_{w \text {. MAX }}$ and the other at $T_{n}=T_{n \text {.MIN }}$ ) of appropriate lengths determined by $z_{1}$.

Figure 7 illustrates the behavior of the minima of cost (Eq. 9) evaluated for different $A$ values for the maximum-singular-minimum profile (full line) as well as for the maximumminimum profile (dotted line).

Corresponding optimal profiles are plotted in Figure 8 for $A$ equal to 0.3 (left plots) and 0.7 (right plots) respectively. Clearly, the performance of the suboptimal maximum-minimum profile is comparable with that of the optimal profile. More specifically, if $A$ is small (equal to 0.3 ), that is, more emphasis is on the conversion efficiency, the cost values for the different profiles practically coincide and the maximumsingular-minimum profile reduces to the maximum-minimum profile. If $A$ is large (for example, equal to 0.7 ), that is, more emphasis on the minimization of global heat loss, the overall cost of the truly optimal bang-singular-bang profile is significantly smaller, although the reactant's conversion with an optimized maximum-minimum profile is slightly better. Remark that for large values of $A$, the maximum-singular-minimum profile reduces to a maximum-singular profile. These observations are summarized in the following result.

Result 3. From a practical point of view, the nearly optimal bang-bang profile is an excellent alternative for the theoretically optimal bang-singular-bang profile.

\section{Conclusions}

At the level of optimal control in time of well mixed reactors, numerous problems have been solved thus far. At the 
level of optimal control in (time and) space of tubular reactors, a rather limited number of solutions to control problems has been reported in literature. In this contribution, it is clearly illustrated that the problem of controlling a steadystate plug-flow reactor -in which a reactant is converted into a desired product - can also be casted within the general optimal control theory framework. In contrast with time varying processes the independent variable is now the space coordinate $z$. The traditional trade-off between process performance and energy consumption is translated into a cost critcrion with terminal and integral parts. The optimal heat exchanger temperature profile for a performance criterion with only terminal cost parts (that is, exit concentration and exit temperature with respect to inlet temperature) is of bang-bang type. This implies that one switches, at most, once between the maximum and minimum value of the heat exchanger temperature.

If integral cost parts are also included (that is, to account for the global heat loss due to high fluid temperatures), it is generically demonstrated that the optimal heat exchanger temperature profile is of bang-singular-bang type. If the integral cost part is not a function of the reactant's concentration, the singular control keeps the reactor temperature constant. For both the maximum-minimum and maximum-singular-minimum profile, it is illustrated that -with an appropriate weighting-an acceptable trade-off between process cfficiency and general heat loss is obtained.

Future work will explore the capability of optimal control theory to optimize and control more realistic tubular reactor processes in which diffusive phenomena are explicitly taken into account, resulting in higher-order PDEs.

\section{Acknowledgment}

Ilse Smets is a research assistant with the Fund for Scientific Research Flanders (FWO). Work supported in part by Projects OT/99/24 and IDO/00/018 of the Research Council of the Katholieke Universiteit Leuven and the Be]gian Program on Interuniversity Poles of Attraction, initiated by the Belgian State, Prime Minister's Office for Science, Technology and Culture. The scientific responsibility is assumed by its authors.

\section{Notation}

$z=$ spatial coordinate. $\mathrm{m}$

$T$ = fluid temperature, $\mathrm{K}$

$T_{\text {in }}=$ inlet fluid temperature, $\mathrm{K}$

$T_{\text {in }}=$ heat exchanger temperature, $\mathrm{K}$

$C=$ reactant concentration, $\mathrm{mol} \cdot \mathrm{L}^{-}$

$C_{\text {in }}=$ inlet reactant concentration, $\mathrm{mol} \cdot \mathrm{L}^{-1}$

$x_{1}=\left(T-T_{\text {in }}\right) / T_{\text {in }}$, dimensionless reactor temperature

$x_{2}=\left(C_{\text {in }}-C\right) / C_{\text {in }}$, dimensionless reactant concentration
$u=\left(T_{x}-T_{\mathrm{in}}\right) / T_{\mathrm{n}}$, dimensionless control variable

$u_{\mathrm{MIN}}=$ lower bound on $u$

$u_{\text {MAX }}=$ upper bound on $u$

$v=$ fluid superficial velocity, $\mathrm{m} \cdot \mathrm{s}^{-1}$

$\Delta H=$ heat of reaction. $\mathrm{cal} \cdot \mathrm{mol}^{-1}$

$C_{p}=$ specific heat, $\mathrm{cal} \cdot \mathrm{kg}^{-1} \cdot \mathrm{K}^{-1}$

$k_{11}=$ kinctic constant, $s^{-1}$

$E=$ activation encrgy, cal $\cdot \mathrm{mol}^{-1}$

$R=$ ideal gas constant, cal $\cdot \mathrm{mol}^{-1} \cdot \mathrm{K}^{-1}$

$h=$ (overall) heat-transfer coefficient, $\mathrm{W} \cdot \mathrm{m}^{-2} \cdot \mathrm{K}^{-1}$

$d=$ reactor diameter, $\mathrm{m}$

$L=$ reactor length, m

$\alpha=k_{0} e^{-E / R T_{i+1}, s^{-i}}$

$\beta=4 h / \rho C_{p} d, s^{-1}$

$\gamma=E / R T_{\text {in }}$

$\delta=-\Delta H C_{\text {in }} / \rho C_{p} T_{\text {in }}$

$\lambda_{1}=$ costate of $x_{1}$

$\lambda_{2}=$ costate of $x_{2}$

$\rho=$ fluid density, $\mathrm{kg} \cdot \mathrm{m}^{-?}$

\section{Literature Cited}

Christofides. P. D., and P. Daoutidis, "Feedback Control of Hyperbolic PDE Systems." AIChE J., 42, 3063 (1996).

Christofides, P. D., and P. Daoutidis, "Robust Control of Hyperbolic PDE Systems," Chem. Eng. Sci., 53, 85 (1998a).

Christofides, P. D., and P. Datoutidis, "Nonlinear Feedback Control of Parabolic PDE Systems," Nonlinear Model Based Process Control, R. Berber and C. Kravaris, eds., NATO ASI series, Kluwer, Dordrecht. The Netherlands (1998b).

Christofides, P. D., Nonlinear and Robust Control of Partial Differential Equation Systems: Methods and Applications to Transport-Reaction Processes. Birkhäuser, Boston (2001).

Dochain, D.. "State Observers for Tubular Reactors with Unknown Kinetics,"J. of Process Control, 10, 259 (2000).

Fjeld, M., and B. Ursin, "Approximate Lumped Models of a Tubular Chemical Reactor, and Their Use in Feedback and Feedforward Control," Proc. IFAC Symp. Multivariable Technical Control Systems, H. Schwarz, ed., North-Holland, Amsterdam (1971).

Gay, D. H., and W. H. Ray. "Identification and Control of Distributed Parameter Systems by Means of the Singular Value Decomposition," Chem. Eng. Sit., 50, 1519 (1995).

Kirk, D. E., Optintal Control Theon: An Introduction, Prentice-Hall, Englewood Cliffs, NJ (1970).

Ray, W. H., "Some Recent Applications of Distributed Parameter Systems Theory-A Survey:" Automatica, 14, 281 (1978).

Ray, W. H., "Advanced Process Control," Butterworths Series in Chemical Engineering, Boston (1981).

Varma, A.. and R. Aris, "Stirred Pots and Empty Tubes," Chenical Reactor Theory, L. Lapidus and N. R. Amundson, eds., PrenticeHall, Englewood Cliffs, NJ, pp. 79-154 (1977).

Winkin, J., D. Dochain, and Ph. Ligarius, "Dynamical Analysis of Distributed Parameter Tubular Reactors," Automatica, 36, 349 (2000).

Ydstic, B. E., and D. P. Coffey, "Distributed Control and Real-Time Optimization of Chemical Processes," Proc. DYCOPS-5, 727 (1998).

Manuscript receiked Dec. 5. 2000, and revision receiced July 27, 2001. 